\title{
Quantum geometry and the Schwarzschild singularity
}

\author{
Abhay Ashtekar ${ }^{1,2,3}$ and Martin Bojowald ${ }^{1,2,3}$ \\ ${ }^{1}$ Physics Department, Institute for Gravitational Physics and Geometry, Pennsylvania State \\ University, University Park, PA 16802, USA \\ ${ }^{2}$ Max-Planck-Institut für Gravitationsphysik, Albert-Einstein-Institut, Am Mühlenberg 1, \\ D-14476 Potsdam, Germany \\ ${ }^{3}$ Isaac Newton Institute for Mathematical Sciences, 20 Clarkson Road, Cambridge CB3 0EH, UK
}

Received 19 September 2005, in final form 24 November 2005

Published 21 December 2005

Online at stacks.iop.org/CQG/23/391

\begin{abstract}
In homogeneous cosmologies, quantum geometry effects lead to a resolution of the classical singularity without having to invoke special boundary conditions at the singularity or introduce $a d$ hoc elements such as unphysical matter. The same effects are shown to lead to a resolution of the Schwarzschild singularity. The resulting quantum extension of spacetime is likely to have significant implications for the black hole evaporation process. Similarities and differences with the situation in quantum geometrodynamics are pointed out.
\end{abstract}

PACS numbers: 04.60.Pp, 04.70.Dy, 04.60.Nc, 03.65.Sq

\section{Introduction}

General relativity provides a subtle and powerful interplay between gravity and geometry, thereby opening numerous possibilities for novel phenomena. Among the most spectacular of the resulting conceptual advances are the predictions that the universe began with a big bang and massive stars can end their lives as black holes. In both cases, one encounters singularities. Spacetime of general relativity literally ends and classical physics comes to a halt.

However, general relativity is incomplete because it ignores quantum effects. It is widely believed that quantum gravity effects become significant in the high curvature regions that develop before singularities are formed. These are likely to significantly change the spacetime structure, making the predictions of general relativity unreliable. Hence, real physics need not stop at the big bang and black hole singularities. While the classical spacetime does end there, quantum spacetime may well extend beyond.

Attempts at extending physics beyond the big-bang singularity date back at least to the 1970s when minisuperspaces were introduced and quantum cosmology was born (see e.g. [1]). These investigations sparked off new developments in different directions ranging from foundations of quantum mechanics to the development of WKB methods to test semiclassicality in quantum cosmology, to the introduction of novel Euclidean methods to calculate 
the appropriate wavefunction of the universe. This deepened our understanding of issues related to the quantum physics of the universe as a whole [2]. However, in these approaches the singularity was not generically resolved. A quantum extension of spacetime required the introduction either of new principles [3], or ad hoc assumptions, such as existence of matter violating energy conditions already at the classical level, or of external clocks that remain insensitive to the infinite curvature encountered at singularities.

Emergence of quantum Riemannian geometry and the associated mathematical techniques [4-6] have provided a new approach to revisit the issue of quantum extensions. Within the minisuperspaces used in quantum cosmology, the big-bang singularity could be resolved without having to introduce external boundary conditions at the big bang, or matter/clocks with unphysical properties [7-9]. In the homogeneous, isotropic minisuperspace coupled to a massless scalar field, in particular, an exhaustive analysis can be carried out [10]. At the analytic level, one can introduce an appropriate inner product on physical states, define Dirac observables and, using the two, construct semi-classical states. One can then use numerical methods to examine the nature of the quantum spacetime. If one begins with a semi-classical state at large late times ('now') and evolves it back in time, it remains semi-classical till one encounters the deep Planck regime near the classical singularity. In this regime, the quantum geometry effects dominate. However, the state becomes semi-classical again on the other side; the deep Planck region serves as a quantum bridge between two large, classical spacetimes. The model is too simple to be applied reliably to the actual universe we live in. However, at a conceptual and mathematical level it demonstrates the new possibilities, systematically made available by quantum geometry.

It is then natural to ask if a similar resolution of singularities occurs also in the context of black holes. Now, there is an extensive literature on the nature of black hole singularities in the classical theory. In particular, detailed mathematical analysis of spherical collapse was carried out by Christodoulou, Dafermos and others. If the collapsing matter is uncharged, the singularity is generically spacelike, as in the Schwarzschild spacetime (see e.g. [11] and references therein). However, if the matter is charged, the generic singularity is not timelike as in the Reissner-Nordstrom case, but null [12]. This is because, as indicated by the early work of Penrose and Simpson and subsequent work of Israel and co-workers, the timelike singularity of the Reissner-Nordstrom solution is unstable. Since we do not have an explicit solution which exhibits the generic null singularity, and since in any case realistic collapse does not involve charged fields, in the spherical case it is appropriate to first focus on spacelike singularities and ask for the nature of quantum spacetime that would result via their resolution ${ }^{4}$. If the singularity is resolved and if the quantum geometry in the deep Planck regime again serves as a bridge to a large classical region beyond, there would be no information loss in the black hole formation and evaporation process [15] (see also [16, 17]). Pure states in the distant past would evolve to pure states in the distant future and one would have a spacetime description of the entire process in a quantum mechanical setting. Thus, a detailed analysis of the fate of black hole singularities is of considerable importance also for the fundamental issue of whether the standard unitary evolution of quantum physics has to be modified in the setting of black holes.

The purpose of this paper is to initiate this investigation using quantum geometry, in the setting of connection dynamics. We will focus just on the minisuperspace that is appropriate

\footnotetext{
4 Moreover, one does not expect quantum gravity effects to resolve all singularities. If, for example, they led to a resolution of the timelike singularity of the negative energy Schwarzschild spacetime, energy would be unbounded below in quantum gravity and the theory would have unphysical features [13]. Rather, one expects that there would be no physical states in quantum gravity that would resemble negative energy Schwarzschild geometry at large distances, whence the issue of 'resolution' would simply not arise.
} 
for describing the geometry interior to the horizon of a Schwarzschild black hole. Since this minisuperspace is spatially homogeneous - of Kantowski-Sachs type-one can take over the techniques that have been developed in the setting of homogeneous cosmologies [8]. Our main result is that the quantum scalar constraint is indeed such that the singularity is resolved. The salient features of our analysis are as follows. First, we use a self-adjoint Hamiltonian constraint. Therefore, our analysis can serve as the point of departure for the construction of the physical Hilbert space either via the group averaging procedure [14] or via deparametrization of the theory as in [10]. Second, we spell out the symmetry reduction procedure that leads to the Kantowski-Sachs minisuperspace within connection dynamics. We will see in sections 3 and 4 that the structure of the resulting phase space is important to the issue of singularity resolution ${ }^{5}$. In particular, this space is an extension of the phase space used in geometrodynamics in that one allows for degenerate triads (and hence 3-metrics). Thanks to this enlargement, points of the reduced phase space corresponding to the singularity do not constitute a boundary, whence the support of the wavefunction 'beyond singularity' can be interpreted geometrically. However, we emphasize that the results of this paper constitute only initial steps for a more complete theory, e.g., along the lines of [10].

Our discussion is organized as follows. In section 2, we discuss the classical theory of the Kantowski-Sachs minisuperspace using connection dynamics. In section 3, we analyse the kinematics of its quantum theory and in section 4 we present quantum dynamics. Section 5 provides a brief summary and directions for future work.

\section{Classical theory}

This section is divided into two parts. In the first, we carry out the Kantowski-Sachs symmetry reduction of vacuum general relativity and in the second we discuss the structure of the resulting phase space.

\subsection{Symmetry reduction}

The portion of the spacetime interior to the horizon of a Schwarzschild black hole can be naturally foliated by 3-manifolds $M$ with topology $\mathbb{R} \times \mathbb{S}^{2}$ such that the geometry on each slice is invariant under the Kantowski-Sachs symmetry group $G=\mathbb{R} \times S O$ (3). In the discussion of this model, we closely follow [9] where the mathematical structure of the simpler, isotropic model is studied in detail.

As in any homogeneous situation, $M$ acquires an equivalence class of positive definite metrics related by an overall constant. We fix one such metric ${ }^{o} q_{a b}$ as well as an orthonormal triad ${ }^{o} e_{i}^{a}$ and a co-triad ${ }^{o} \omega_{a}^{i}$, compatible with it. With the given symmetry, we choose the metric on the orbits of the $S O(3)$ action to be the unit 2-sphere metric, $\mathrm{d} \vartheta^{2}+\sin ^{2} \vartheta \mathrm{d} \varphi^{2}$ in polar coordinates $\vartheta, \varphi$. Locally, the corresponding co-triad elements $\mathrm{d} \vartheta$ and $\sin \vartheta \mathrm{d} \varphi$ can be completed to an orthonormal co-triad by a third form $\alpha$. Thanks to the Maurer-Cartan relations for the symmetry group, $\alpha$ must be closed and therefore exact on the given topology, whence it

5 A quantization of the Kantowski-Sachs minisuperspace is available in the geometrodynamical framework [18]. Unfortunately, it does not shed light on singularity resolution because the location of the singularity in the minisuperspace was misidentified. In the notation used in [18], the singularity lies at $(a=\infty, b=0)$-not at ( $a=0, b=0$ ) as assumed there. Therefore, neither is the inverse volume operator (17) of [18] bounded or well defined at the singularity nor does the discrete equation (23) of [18] enable one to evolve across the singularity.

Note added: after our paper appeared on the archives, this misidentification was corrected in a revised version of [18]. However, since the classical singularity lies at $a=\infty$, the arguments for its removal continue to remain unclear. 
defines a third adapted coordinate $x$ via $\alpha=\mathrm{d} x$. In these coordinates, the background metric has line element

$$
\mathrm{d} s_{o}^{2}=\mathrm{d} x^{2}+\mathrm{d} \vartheta^{2}+\sin ^{2} \vartheta \mathrm{d} \varphi^{2}
$$

and determinant ${ }^{o} q=\sin ^{2} \vartheta$.

In connection dynamics, the canonically conjugate pair consists of fields $\left(A_{a}^{i}, E_{i}^{a}\right)$ of an $S U$ (2) connection 1-form $A_{a}^{i}$ and a (possibly degenerate) triad $E_{i}^{a}$ of density weight 1 on $M$. This pair is invariant under the symmetry group if it satisfies

$$
\mathcal{L}_{\xi} A=D \Lambda \quad \text { and } \quad \mathcal{L}_{\xi} E=[E, \Lambda]
$$

for any symmetry vector field $\xi^{a}$ and some generator $\Lambda^{i}$ of local $S U(2)$ gauge transformations (which may depend on $\xi^{a}$ ). One can verify that any such symmetric pair is gauge equivalent to fields of the form ${ }^{6}$ :

$$
\begin{aligned}
& A=\tilde{c} \tau_{3} \mathrm{~d} x+\left(\tilde{a} \tau_{1}+\tilde{b} \tau_{2}\right) \mathrm{d} \vartheta+\left(-\tilde{b} \tau_{1}+\tilde{a} \tau_{2}\right) \sin \vartheta \mathrm{d} \varphi+\tau_{3} \cos \vartheta \mathrm{d} \varphi \\
& E=\tilde{p}_{c} \tau_{3} \sin \vartheta \frac{\partial}{\partial x}+\left(\tilde{p}_{a} \tau_{1}+\tilde{p}_{b} \tau_{2}\right) \sin \vartheta \frac{\partial}{\partial \vartheta}+\left(-\tilde{p}_{b} \tau_{1}+\tilde{p}_{a} \tau_{2}\right) \frac{\partial}{\partial \varphi}
\end{aligned}
$$

where $\tilde{a}, \tilde{b}, \tilde{c}$ are real constants and $\tau^{j}$ is the standard basis in $s u(2)$, satisfying $\left[\tau_{i}, \tau_{j}\right]=\epsilon_{i j}{ }^{k} \tau_{k}$. However, this does not exhaust the freedom to perform local $S U(2)$ gauge transformations entirely. There is still the freedom to perform a global $S U(2)$ transformation along $\tau_{3}$ which rotates the 'vectors' ( $\tilde{a}, \tilde{b})$ and $\left(\tilde{p_{a}}, \tilde{p_{b}}\right)$. We will return to this freedom in the next subsection.

From the invariant triad density (3), we can derive the corresponding co-triad $\omega$ :

$$
\omega=\omega_{c} \tau_{3} d x+\left(\omega_{a} \tau_{1}+\omega_{b} \tau_{2}\right) d \vartheta+\left(-\omega_{b} \tau_{1}+\omega_{a} \tau_{2}\right) \sin \vartheta \mathrm{d} \varphi
$$

where

$$
\omega_{c}=\frac{\operatorname{sgn} \tilde{p}_{c} \sqrt{\tilde{p}_{a}^{2}+\tilde{p}_{b}^{2}}}{\sqrt{\left|\tilde{p}_{c}\right|}}, \quad \omega_{b}=\frac{\sqrt{\left|\tilde{p}_{c}\right|} \tilde{p}_{b}}{\sqrt{\tilde{p}_{a}^{2}+\tilde{p}_{b}^{2}}} \quad \text { and } \quad \omega_{a}=\frac{\sqrt{\left|\tilde{p}_{c}\right|} \tilde{p}_{a}}{\sqrt{\tilde{p}_{a}^{2}+\tilde{p}_{b}^{2}}} \text {. }
$$

The co-triad in turn determines its spin connection, the $s u(2)$-valued 1-form $\Gamma$. As one might expect from the structure of the triad, $\Gamma$ turns out to be the standard 'magnetic monopole' connection:

$$
\Gamma=\cos \vartheta \tau_{3} \mathrm{~d} \varphi
$$

Consequently, the $s u(2)$-valued extrinsic curvature 1 -form $K$ is given by

$$
\gamma K:=A-\Gamma=\tilde{c} \tau_{3} \mathrm{~d} x+\left(\tilde{a} \tau_{1}+\tilde{b} \tau_{2}\right) \mathrm{d} \vartheta+\left(-\tilde{b} \tau_{1}+\tilde{a} \tau_{2}\right) \sin \vartheta \mathrm{d} \varphi .
$$

Finally, the curvature $F_{a b}$ of $A_{a}$ can easily be computed. Its dual, the $s u(2)$-valued vector density $B^{a}:=\frac{1}{2} \eta^{a b c} F_{b c}$ has the same invariant form as $E^{a}$ :

$B=\left(\tilde{a}^{2}+\tilde{b}^{2}-1\right) \tau_{3} \sin \vartheta \frac{\partial}{\partial x}+\left(\tilde{a} \tau_{1}+\tilde{b} \tau_{2}\right) \tilde{c} \sin \vartheta \frac{\partial}{\partial \vartheta}+\left(-\tilde{b} \tau_{1}+\tilde{a} \tau_{2}\right) \tilde{c} \frac{\partial}{\partial \varphi}$.

These expressions will be used in sections 3 and 4 .

6 More precisely, invariant connections [19, 20] carry a non-negative, integer-valued topological charge which we have set equal to 1 in the above expressions. (In general, it would multiply the last contribution to (2); see e.g. [21].) Each value of this charge gives rise to an independent sector of invariant connections. However, only the sector used here allows non-degenerate triads invariant under (1) with the same local gauge transformation in $A$ and $E$. The fact that $A$ in (2) satisfies (1) can be verified as follows. If we denote the three generators of the $S O$ (3) action on $M$ by $X=\sin \varphi \partial_{\vartheta}+\cot \vartheta \cos \varphi \partial_{\varphi}, Y=-\cos \varphi \partial_{\vartheta}+\cot \vartheta \sin \varphi \partial_{\varphi}$ and $Z=\partial_{\varphi}$, and the generator of the $\mathbb{R}$-action by $t^{a}$, then $\mathcal{L}_{X} A=D(\cos \varphi / \sin \vartheta) \tau_{3}, \mathcal{L}_{Y} A=D(\sin \varphi / \sin \vartheta) \tau_{3}, \mathcal{L}_{Z} A=0$ and $\mathcal{L}_{t} A=0$. Invariance of $E$ in (3) follows analogously or by noting that $E_{i}^{a} \delta A_{a}^{i}$ defines a gauge invariant and (spatially) constant 1-form on phase space. 


\subsection{The reduced phase space}

The symmetry reduction procedure led us to a six-dimensional reduced phase space $\tilde{\Gamma}$ with coordinates $\left(\tilde{a}, \tilde{b}, \tilde{c} ; \tilde{p}_{a}, \tilde{p}_{b}, \tilde{p}_{c}\right)$. Let us begin by computing the symplectic structure $\tilde{\Omega}$ on it. The basic idea, as in all symmetry reductions, is to pull back the symplectic structure of the full theory to the symmetry reduced phase space. However, since the full symplectic structure involves an integral over $M$ and since the fields of interest are homogeneous also in the non-compact $\mathbb{R}$-direction, as usual, we are led to consider only a finite interval $\mathcal{I}$ in the $\mathbb{R}$-direction. Let the length of this interval (w.r.t. the fiducial metric ${ }^{o} q_{a b}$ ) be $L_{o}$. Then, the symplectic structure on the reduced phase space is given by

$$
\tilde{\boldsymbol{\Omega}}=\frac{L_{o}}{2 \gamma G}\left(2 \mathrm{~d} \tilde{a} \wedge \mathrm{d} \tilde{p}_{a}+2 \mathrm{~d} \tilde{b} \wedge \mathrm{d} \tilde{p}_{b}+\mathrm{d} \tilde{c} \wedge \mathrm{d} \tilde{p}_{c}\right),
$$

where $\gamma$ is the Barbero-Immirzi parameter and $G$ is the gravitational constant. (The volume of the $\mathbb{S}^{2}$ orbits of the $S O(3)$ action, defined by the fiducial metric, is the standard one, $4 \pi$.)

Because of the forms (2) and (3) of invariant connections and triads, the vector constraint is automatically satisfied. However, because of the residual, global $S U(2)$ gauge freedom mentioned in section 2.1, the Gauss constraint is not. Inserting the invariant connection and triad into the Gauss constraint, we obtain

$$
\mathcal{C}_{\text {Gauss }}=\tilde{a} \tilde{p}_{b}-\tilde{b} \tilde{p}_{a}=0,
$$

which generates simultaneous rotations of the pairs $(\tilde{a}, \tilde{b})$ and $\left(\tilde{p}_{a}, \tilde{p}_{b}\right)$. Thus, only the 'norms' $\sqrt{\tilde{a}^{2}+\tilde{b}^{2}}, \sqrt{\tilde{p}_{a}^{2}+\tilde{p}_{b}^{2}}$ and the 'scalar product' $\left(\tilde{a} \tilde{p}_{a}+\tilde{b} \tilde{p}_{b}\right)$ are gauge invariant. We will fix this gauge freedom as follows. If $(\tilde{a}, \tilde{b})=0$, we rotate the triad components such that $\tilde{p}_{a}=0$. Otherwise we rotate $(\tilde{a}, \tilde{b})$ such that $\tilde{a}=0$ which implies $\tilde{b} \neq 0$. Then, $\mathcal{C}_{\text {Gauss }}=0$ implies $\tilde{p}_{a}=0$. There is still a residual gauge freedom $\Pi_{b}:\left(b, p_{b}\right) \rightarrow\left(-b,-p_{b}\right)$ which changes the signs of $b$ and $p_{b}$ simultaneously. This is just the parity transformation in the $p_{b}$ variable. One can either retain this freedom and ensure that all the final constructions are invariant with respect to this ' $b$-reflection' or eliminate it by a gauge choice such as $\tilde{p}_{b} \geqslant 0$. This gauge choice turns out to be particularly convenient for classical dynamics. In the quantum theory, on the other hand, it is more natural to retain the freedom at first and then ask that physical states be invariant under the parity operator $\hat{\Pi}_{b}$ implementing this transformation. Therefore, we will allow both possibilities. In either case, the four-dimensional phase space carries a global chart $\left(\tilde{b}, \tilde{c} ; \tilde{p}_{b}, \tilde{p}_{c}\right)$ (where $\tilde{p}_{b} \geqslant 0$ if the ' $b$-parity gauge' is fixed). These coordinates are subject to the scalar or the Hamiltonian constraint, discussed below.

We first note that these variables and, because of its explicit dependence on $L_{o}$, the symplectic structure $\tilde{\boldsymbol{\Omega}}$ depend on the fiducial metric. It is convenient to remove this dependence by rescaling the variables in a manner that is motivated by their scaling properties,

$$
(b, c):=\left(\tilde{b}, L_{o} \tilde{c}\right), \quad\left(p_{b}, p_{c}\right):=\left(L_{o} \tilde{p}_{b}, \tilde{p}_{c}\right) .
$$

We now have a one-to-one parametrization of the gauge fixed phase space $\Gamma$ by $\left(b, p_{b}, c, p_{c}\right)$ with symplectic structure

$$
\boldsymbol{\Omega}=\frac{1}{2 \gamma G}\left(2 \mathrm{~d} b \wedge \mathrm{d} p_{b}+\mathrm{d} c \wedge \mathrm{d} p_{c}\right) .
$$

For later purposes let us express the volume of our elementary cell $\mathcal{I} \times \mathbb{S}^{2}$ and areas bounded by our preferred family of curves as functions on the reduced phase space. The volume is given by

$$
V=\int \mathrm{d}^{3} x \sqrt{|\operatorname{det} E|}=4 \pi L_{o} \sqrt{\left|\tilde{p}_{c}\right|}\left|\tilde{p}_{b}\right|=4 \pi \sqrt{\left|p_{c}\right|}\left|p_{b}\right| .
$$


The three surfaces $S_{x, \varphi}, S_{x, \vartheta}$ and $S_{\vartheta, \varphi}$ of interest are, respectively, bounded by the interval $\mathcal{I}$ and the equator, $\mathcal{I}$ and a great circle along a longitude, and the equator and a longitude (so that $S_{\vartheta, \varphi}$ forms a quarter of the sphere $\mathbb{S}^{2}$ ). Their areas are given by

$$
A_{x, \vartheta}=2 \pi\left|p_{b}\right|, \quad A_{x, \varphi}=2 \pi\left|p_{b}\right| \quad \text { and } \quad A_{\vartheta, \varphi}=\pi\left|p_{c}\right| .
$$

Finally, let us consider the Hamiltonian constraint and classical dynamics. On the reduced phase space $\Gamma$, the constraint functional of the full theory

$$
\mathcal{C}_{\text {Ham }}=\int \mathrm{d} x^{3} N e^{-1}\left[\epsilon_{i j k} F_{a b}^{i} E_{j}^{a} E_{k}^{b}-2\left(1+\gamma^{2}\right) K_{[a}^{i} K_{b]}^{j} E_{i}^{a} E_{j}^{b}\right],
$$

where $e:=\sqrt{|\operatorname{det} E|} \operatorname{sgn}(\operatorname{det} E)$, reduces to

$$
\mathcal{C}_{\text {Ham }}=-\frac{8 \pi N}{\gamma^{2}} \frac{\operatorname{sgn} p_{c}}{\sqrt{\left|p_{c}\right|} p_{b}}\left[\left(b^{2}+\gamma^{2}\right) p_{b}^{2}+2 c p_{c} b p_{b}\right],
$$

where, as is usual in the homogeneous models, we have chosen a constant lapse function $N$. To simplify the equations of motion, it is convenient ${ }^{7}$ to choose $N=\frac{\gamma \operatorname{sgn} p_{c} \sqrt{\left|p_{c}\right|}}{16 \pi G b}$. Then the Hamiltonian constraint becomes $\mathcal{C}_{\text {Ham }}:=-(2 \gamma G)^{-1}\left[\left(b^{2}+\gamma^{2}\right) p_{b} / b+2 c p_{c}\right]$, yielding the following equations of motion:

$$
\begin{array}{ll}
\dot{b}=-\frac{1}{2}\left(b+\gamma^{2} b^{-1}\right), & \dot{p}_{b}=\frac{1}{2}\left(p_{b}-\gamma^{2} b^{-2} p_{b}\right), \\
\dot{c}=-2 c, & \dot{p}_{c}=2 p_{c},
\end{array}
$$

where the 'dot' denotes derivative with respect to the affine parameter of the Hamiltonian vector field. The equations for $\left(c, p_{c}\right)$ can easily be solved by $c(T)=c_{0} \mathrm{e}^{-2 T}$ and $p_{c}(T)=p_{c}^{(0)} \mathrm{e}^{2 T}$. For the other components we obtain $b(T)= \pm \gamma \sqrt{\mathrm{e}^{-\left(T-T_{0}\right)}-1}$ for $b$ and, using this result, $p_{b}(T)=p_{b}^{(0)} \sqrt{\mathrm{e}^{T+T_{0}}-\mathrm{e}^{2 T}}$. If we introduce the new time parameter $t:=\mathrm{e}^{T}$ and the constant $m:=\frac{1}{2} \mathrm{e}^{T_{0}}$, this gives

$$
\begin{aligned}
b(t) & = \pm \gamma \sqrt{(2 m-t) / t}, & & p_{b}(t)=p_{b}^{(0)} \sqrt{t(2 m-t)}, \\
c(t) & =\mp \gamma m p_{b}^{o} t^{-2}, & & p_{c}(t)= \pm t^{2},
\end{aligned}
$$

where we have fixed the multiplicative constant in the expressions of $p_{c}(t)$ and $c(t)$, respectively, by requiring that $\left|p_{c}\right|$ be the geometric radius of the 2-sphere orbits of the $S O$ (3) symmetry and by using the Hamiltonian constraint. Projections to the $p_{b}-p_{c}$ plane of typical trajectories are shown in figure 1 . It is clear from the figure that $p_{c}$ can be taken to be the 'internal time parameter'. In section 4.1, this interpretation will let us regard the quantum Hamiltonian constraint as an 'evolution equation' with respect to an internal time parameter defined by the eigenvalues of the operator $\hat{p}_{c}$.

Note that we have a two-parameter family of solutions, labelled by $m$ and $p_{b}^{(0)}$, as one would expect from the fact that the reduced phase space is two dimensional. However, in a spacetime description we expect only a one-parameter family, labelled by $m$. This discrepancy can be traced back to a standard tension between the Hamiltonian and spacetime notions of gauge (for a detailed discussion in the context of Bianchi models, see [22], and in spherically symmetric models, $[23,24])$. In the Hamiltonian description, rescalings of $p_{b}^{(0)}$ do not correspond to gauge because they are not generated by any constraint. In the spacetime description, on the other hand, they can be absorbed by a rescaling of the coordinate $x$. Therefore, to make contact

7 With this choice, the points $b=0$ or $p_{c}=0$ have to be excised in the discussion of dynamics. The explicit form of the solutions below shows that this is not overly restrictive since on classical solutions these points correspond to the singularity or the horizon. 


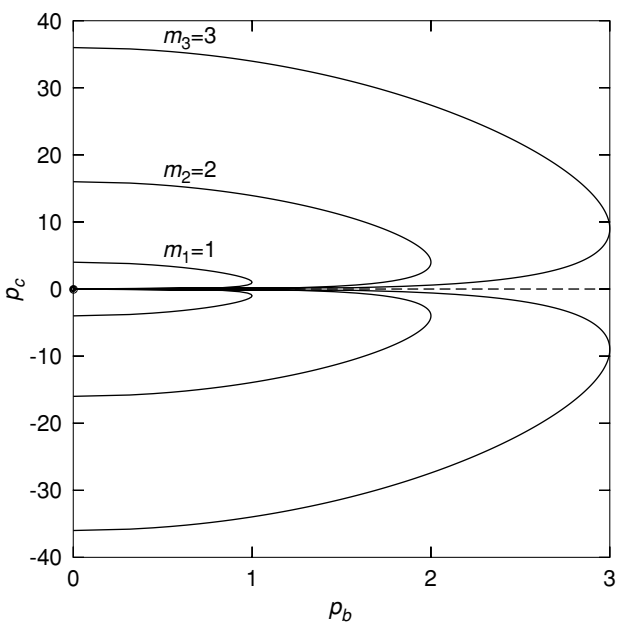

Figure 1. Dynamical trajectories in the $p_{b}-p_{c}$ plane. Each trajectory reaches the maximum value $m$ of $p_{b}$ and meets the $p_{c}=0$ axis only at the point where $p_{b}$ also vanishes (i.e., at the 'origin'). Solutions related by the reflection symmetry $p_{c} \rightarrow-p_{c}$ define the same metric but carry triads of opposite orientation. For simplicity, the ' $b$-parity gauge' is fixed by requiring $p_{b} \geqslant 0$.

with the spacetime description, let us fix $p_{b}^{(0)}=1$. Then, the above solution to the evolution equations defines a one-parameter family of 3-metrics:

$$
q_{a b}(t)=\left(\frac{2 m}{t}-1\right) \nabla_{a} x \nabla_{b} x+t^{2}\left(\nabla_{a} \vartheta \nabla_{b} \vartheta+\sin ^{2} \vartheta \nabla_{a} \varphi \nabla_{b} \varphi\right)
$$

defining precisely the Schwarzschild solution of mass $m$, interior to the horizon. It will be useful to note that, in this solution $p_{c}>0, p_{b}=0$ at the horizon $(t=2 m)$ and $p_{c}=0$ and $b, c$ diverge at the singularity.

We will see in the next section that it is convenient to regard the space $\mathcal{M}$ spanned by $p_{b}$ and $p_{c}$ as the minisuperspace on which quantum wavefunctions are defined. (In that discussion, we will not fix the ' $b$-parity gauge'; so $p_{c}$ and $p_{b}$ take values on the entire real line.) Let us therefore interpret various regions in $\mathcal{M}$. The triad is non-degenerate everywhere except when $p_{b}=0$ or $p_{c}=0$. However, the two degeneracies are of very different geometric and physical origin. Geometrically (i.e., independent of classical dynamics), $p_{c}=0$ separates two regions with triads of opposite orientations, given by $\operatorname{sgn} \operatorname{det} E=\operatorname{sgn}\left(p_{c} p_{b}^{2}\right)$. On the other hand, as (4) (together with our gauge choice which ensures $a=p_{a}=0$ ) shows, the orientation would not have changed even if we had extended $\mathcal{M}$ across $p_{b}=0$, allowing for negative values for $p_{b}$. More importantly, while the co-triad (4) remains smooth and only becomes degenerate along $p_{b}=0$, it diverges along $p_{c}=0$, signalling the classical singularity. This suggests that the line $p_{b}=0$ corresponds to the horizon and $p_{c}=0$ to the singularity. This is confirmed from physical considerations involving classical dynamics. Classical solutions can be smoothly extended to the line $p_{b}=0$ which is reached for $t=2 \mathrm{~m}$. The curvature $B$ remains well defined there (see (8)). The other line, $p_{c}=0$, is very different. Along classical solutions, the line $p_{c}=0$ is approached as $t \rightarrow 0$, whence the components (and invariants) of curvature $B$ also diverge. This line corresponds to the singularity and cannot be crossed by any classical dynamical trajectory. Finally, note that since the line $p_{c}=0$ separates the two regions of $\mathcal{M}$, where only the triad orientation is reversed, the two get identified in geometrodynamics. Therefore, the singularity would lie at a boundary of the minisuperspace in geometrodynamics, making the geometrical meaning of 'evolution across 
the singularity' obscure. Furthermore, since the Arnowitt-Deser-Misner (ADM) variables are based on covariant metrics (rather than contravariant triad densities), as in other homogeneous models [8], one of the ADM variables becomes infinite at the classical singularity. This further complicates a discussion of the singularity structure and its resolution.

\section{Quantum kinematics}

This section is divided into two parts. In the first, we introduce the basic quantization procedure and in the second we discuss quantum geometry with emphasis on the classical singularity.

\subsection{Basics}

As in the isotropic model [9], we will follow the general procedure used in the full theory [4-6]. Thus, the elementary configuration variables will be given by holonomies along curves in $M$ and the momenta by fluxes of triads along 2-surfaces in $M$. However, because of symmetry reduction, one need not consider all (piecewise analytic) curves or surfaces; a judiciously chosen, smaller subset suffices to obtain a set of functions that is sufficiently large to separate points of the reduced phase space.

Let us begin with the holonomies. We will restrict ourselves to three sets of curves: those along the $\mathbb{R}$ direction of $M$ with oriented length $\tau L_{o}$, those along the equator of $\mathbb{S}^{2}$ with oriented length $\mu$ and those along the longitudes of $\mathbb{S}^{2}$ also with oriented length $\mu$, where all lengths and orientations are defined using the fiducial triad. (Thus, $\tau, \mu$ are positive if the tangents to the respective curves are parallel to the triad vectors and negative if they are anti-parallel.) Holonomies along these curves suffice to completely determine any invariant connection $(2)^{8}$ :

$$
\begin{aligned}
& h_{x}^{(\tau)}(A)=\exp \int_{0}^{\tau L_{o}} \mathrm{~d} x \tilde{c} \tau_{3}=\cos \frac{\tau c}{2}+2 \tau_{3} \sin \frac{\tau c}{2}, \\
& h_{\varphi}^{(\mu)}(A)=\exp -\int_{0}^{\mu} \mathrm{d} \varphi \tilde{b} \tau_{1}=\cos \frac{\mu b}{2}-2 \tau_{1} \sin \frac{\mu b}{2}, \\
& h_{\vartheta}^{(\mu)}(A)=\exp \int_{0}^{\mu} \mathrm{d} \vartheta \tilde{b} \tau_{2}=\cos \frac{\mu b}{2}+2 \tau_{2} \sin \frac{\mu b}{2} .
\end{aligned}
$$

Matrix elements of these holonomies are functions of the reduced connection and constitute our configuration variables. Elements of the algebra they generate are almost periodic functions of $b$ and $c$ of the form $f(b, c)=\sum_{\mu, \tau} f_{\mu \tau} \exp \frac{\mathrm{i}}{2}(\mu b+\tau c)$, where $f_{\mu, \tau} \in \mathbb{C}, \mu, \tau \in \mathbb{R}$ and the sum extends over a finite set. This algebra is the Kantowski-Sachs analogue of the algebra of cylindrical functions in the full theory [4-6]. We will therefore denote it by $\mathrm{Cyl}_{\mathrm{KS}}$. Consider the $C^{\star}$ algebra obtained by completing $\mathrm{Cyl}_{\mathrm{KS}}$ using the sup norm. The quantum configuration space $\overline{\mathcal{A}}_{\mathrm{KS}}$ is the Gel'fand spectrum of this algebra. From the structure of the algebra, it follows that the spectrum is naturally isomorphic to the Bohr compactification $\overline{\mathbb{R}}_{\text {Bohr }}^{2}$ of the Abelian group $\mathbb{R}^{2}$ [26, 27]. (Recall that in the isotropic case [9], the quantum configuration space is isomorphic to $\overline{\mathbb{R}}_{\mathrm{Bohr}}$.) The Hilbert space $\tilde{\mathcal{H}}$ is obtained by the Cauchy completion of $\mathrm{Cyl}_{\mathrm{KS}}$ with respect to the natural Haar measure $\mu_{o}$ on the Abelian group $\overline{\mathbb{R}}_{\text {Bohr }}^{2} ; \tilde{\mathcal{H}}=L^{2}\left(\overline{\mathbb{R}}_{\text {Bohr }}^{2}, \mathrm{~d} \mu_{o}\right)$. Employing the standard bra-ket notation, we can define a basis $|\mu, \tau\rangle$ in $\tilde{\mathcal{H}}$ via

$$
\langle b, c \mid \mu, \tau\rangle=\exp \left(\frac{\mathrm{i}}{2}(\mu b+\tau c)\right), \quad \mu, \tau \in \mathbb{R} .
$$

8 In the fully gauge fixed setting, we could omit $h_{\vartheta}$ since it is related to $h_{\varphi}$ by conjugation with $\exp \tau_{3}$. However, we will use a more 'democratic' approach which is also applicable when $a$ is not made to vanish by gauge fixing. 
This is an orthonormal basis:

$$
\left\langle\mu^{\prime}, \tau^{\prime} \mid \mu, \tau\right\rangle=\delta_{\mu^{\prime} \mu} \delta_{\tau^{\prime} \tau},
$$

where, on the right-hand side, we have the Kronecker symbol, rather than the Dirac delta distribution. Thus, each basis vector is normalizable and has unit norm.

As one would expect, the configuration-i.e., holonomy-operators $\hat{h}_{x}^{(\tau)}, \hat{h}_{\varphi}^{(\mu)}, \hat{h}_{\vartheta}^{(\mu)}$ operate on $\tilde{\mathcal{H}}$ by multiplication. However, as in the full theory, these operators are not required to be weakly continuous in parameters $\mu$ and $\tau$, whence there are no operators $\hat{b}, \hat{c}$ corresponding to the connection itself. Consequently, although we have only a finite number of degrees of freedom, the von-Neumann uniqueness theorem is inapplicable and this quantum theory is inequivalent to a standard 'Schrödinger quantization' (for a further discussion, see $[26,27])$. We will see that, as in the isotropic model [9], this inequivalence has important consequences.

For the momentum operators, we consider fluxes of triads along preferred 2-surfaces. Apart from fixed kinematical factors, they are given by components $p_{b}, p_{c}$ of triads which, in view of the symplectic structure (12), are represented by operators ${ }^{9}$

$$
\hat{p}_{b}=-\mathrm{i} \gamma \ell_{\mathrm{Pl}}^{2} \frac{\partial}{\partial b}, \quad \hat{p}_{c}=-2 \mathrm{i} \gamma \ell_{\mathrm{Pl}}^{2} \frac{\partial}{\partial c} .
$$

Their eigenstates are the basis states (23),

$$
\hat{p}_{b}|\mu, \tau\rangle=\frac{1}{2} \gamma \ell_{\mathrm{Pl}}^{2} \mu|\mu, \tau\rangle, \quad \hat{p}_{c}|\mu, \tau\rangle=\gamma \ell_{\mathrm{Pl}}^{2} \tau|\mu, \tau\rangle .
$$

However, we still have to incorporate the residual gauge freedom which corresponds to a parity reflection in the $b$ degree of freedom. Therefore, only those states in $\tilde{\mathcal{H}}$ which are invariant under the parity operator $\hat{\Pi}_{b}:|\mu, \tau\rangle \rightarrow|-\mu, \tau\rangle$ can belong to the kinematical Hilbert space $\mathcal{H}$. A basis in $\mathcal{H}$ is thus given by

$$
\frac{1}{\sqrt{2}}[|\mu, \tau\rangle+|-\mu, \tau\rangle]
$$

Finally, we express the volume operator in terms of triad operators $\hat{p}_{b}, \hat{p}_{c}$. Recall that the region of $M$ under consideration is of the type $\mathcal{I} \times \mathbb{S}^{2}$, where $\mathcal{I}$ has length $L_{o}$ with respect to the fiducial metric. From the classical expression (13) of the volume $V$ of this region, it follows that the operator $\hat{V}$ is given by $\hat{V}=4 \pi\left|\hat{p}_{b}\right| \sqrt{\left|\hat{p}_{c}\right|}$. It is diagonal in our $|\mu, \tau\rangle$ basis and the eigenvalues are

$$
V_{\mu \tau}=2 \pi \gamma^{3 / 2}|\mu| \sqrt{|\tau|} \ell_{\mathrm{Pl}}^{3} .
$$

As in the isotropic case, the volume of our cell $\mathcal{I} \times \mathbb{S}^{2}$ with respect to the fiducial metric can be large, but its volume in the 'elementary' state exp $\frac{i}{2}(b+c)$ is of Planck size.

\subsection{Quantum geometry}

As in the isotropic case, the triad operators $\hat{p}_{b}, \hat{p}_{c}$ commute whence, in contrast to the full theory, one can construct the triad representation. In this subsection, we will study the quantum Riemannian geometry using this representation. In the next subsection, we will see that the representation is also convenient for dynamics, as it suggests an intuitive interpretation for the action of the Hamiltonian constraint.

Let us then expand a general state in terms of eigenstates of the triad operators, $|\psi\rangle=\sum_{\mu \tau} \psi_{\mu \tau}|\mu, \tau\rangle$, and use the coefficients $\psi_{\mu \tau}$ to represent the state. This wavefunction

9 In this paper, we use the standard quantum gravity convention, $\ell_{\mathrm{Pl}}^{2}=G \hbar$. Unfortunately, this is different from the convention $\ell_{\mathrm{Pl}}^{2}=8 \pi G \hbar$ used in most of the loop quantum cosmology literature. Hence, care should be taken while comparing detailed numerical factors. 
$\psi_{\mu \tau}$ is supported on the minisuperspace $\mathcal{M}$ coordinatized by $p_{b}$ and $p_{c}$. In the classical theory, already at the kinematical level, the lines $p_{b}=0$ and $p_{c}=0$ are special: the co-triad (4) becomes degenerate along $p_{b}=0$ (and the line represents the horizon) while it diverges on the line $p_{c}=0$ (and this line represents the singularity). It is then natural to examine wavefunctions $\psi_{\mu \tau}$ which are supported on lines $\mu=0$ and $\tau=0$ and ask, already at the kinematical level, for the nature of quantum geometry they represent.

Let us consider the co-triad component $\omega_{c} \equiv \operatorname{sgn}\left(p_{c}\right)\left|p_{b}\right| / \sqrt{\left|p_{c}\right|}$ along $\mathrm{d} x$ (see (4)). Since it involves an inverse power of $p_{c}$ and since there exist normalizable kets $|\mu, \tau=0\rangle$ of the operator $\hat{p}_{c}$ with zero eigenvalue, the naive operator obtained by replacing $p_{b}$ and $p_{c}$ by corresponding operators is not even densely defined, let alone self-adjoint. To define $\hat{\omega}_{c}$, therefore, a new strategy is needed. We will follow what is by now a standard procedure, adapted from Thiemann's analysis in the full theory [25]. The first step is to express $\omega_{c}$ in terms of the elementary variables which do have unambiguous quantum analogues-holonomies and positive powers of $p_{b}, p_{c}$-and Poisson brackets between them. On the classical phase space, we have the exact equality:

$$
\omega_{c}=\frac{1}{2 \pi \gamma G} \operatorname{Tr}\left(\tau_{3} h_{x}\left\{h_{x}^{-1}, V\right\}\right),
$$

where $h_{x}$ is the holonomy along the interval $\mathcal{I}$, i.e., along the edge of length $L_{o}$ (with respect to the fiducial metric) we fixed in our construction of the phase space. Then, replacing $h_{x}$ and $V$ by their unambiguous quantum analogues and the Poisson bracket by $1 / \mathrm{i} \hbar$ times the commutator, we obtain

$$
\begin{aligned}
\hat{\omega}_{c} & =-\frac{\mathrm{i}}{2 \pi \gamma \ell_{\mathrm{Pl}}^{2}} \operatorname{Tr}\left(\tau_{3} \hat{h}_{x}\left[\hat{h}_{x}^{-1}, \hat{V}\right]\right) \\
& =-\frac{\mathrm{i}}{2 \pi \gamma \ell_{\mathrm{Pl}}^{2}}\left(\sin \frac{c}{2} \hat{V} \cos \frac{c}{2}-\cos \frac{c}{2} \hat{V} \sin \frac{c}{2}\right) .
\end{aligned}
$$

It turns out that this operator is diagonalized by our basis $|\mu, \tau\rangle$ of (23),

$\hat{\omega}_{c}|\mu, \tau\rangle=\frac{1}{4 \pi \gamma \ell_{\mathrm{Pl}}^{2}}\left(V_{\mu, \tau+1}-V_{\mu, \tau-1}\right)|\mu, \tau\rangle=\frac{\sqrt{\gamma}}{2} \ell_{\mathrm{Pl}}|\mu|(\sqrt{|\tau+1|}-\sqrt{|\tau-1|})|\mu, \tau\rangle$

For large $\tau$, far away from the classical singularity, these eigenvalues are very close to the classical expectation $\left|\omega_{c}\right|=\left|p_{b}\right| / \sqrt{\left|p_{c}\right|}$. This is even true for small $\mu$, i.e. we do not need to be far from the horizon. Closer to the classical singularity $p_{c}=0$, on the other hand, the behaviour becomes significantly different from the classical one. At the classical singularity itself, where $\left|p_{b}\right| / \sqrt{\left|p_{c}\right|}$ diverges, the eigenvalue of $\hat{\omega}_{c}$ is zero $^{10}$.

Remark. In the above definition of $\hat{\omega}_{c}$, we needed a holonomy in the $x$-direction. We chose to evaluate it using the interval $\mathcal{I}$ of length $L_{o}$ with respect to the fiducial metric. While this choice is natural because this interval appears already in the construction of the phase space, we could have used an interval of length $L_{o} \delta$ for some $\delta$. Then the resulting operator would also have been bounded on the entire Hilbert space $\tilde{\mathcal{H}}$, and $|\mu, \tau=0\rangle$ would again have been eigenvectors of the operator with zero eigenvalue. Thus, the qualitative properties of the operator are insensitive to $\delta$. Furthermore, from general considerations one can argue [9] that $\delta$ should be of the order of 1 . However, the value of the upper bound of the spectrum does depend on $\delta$. Therefore, as in the isotropic model, the precise numerical coefficient in this bound should not be attributed physical significance.

\footnotetext{
${ }^{10}$ Exact classical identities such as (29) for objects containing inverse powers are available only in some reduced models. More generally, the bracket between holonomies and volume is more complicated [29]. These complications lead to additional correction terms and the resulting operator is no longer bounded [30] on eigenspaces of $\hat{p}_{b}$.
} 
Let us now turn to the second triad component $\omega_{b}=\operatorname{sgn} p_{b} \sqrt{\left|p_{c}\right|}$. Since $\sqrt{|\tau|}$ is a well-defined function of $\tau$ on the entire spectrum of $\hat{p}_{c}$, we can quantize $\omega_{b}$ directly. This operator is again diagonal in our basis $|\mu, \tau\rangle$ and its eigenvalues are given by

$$
\left.\hat{\omega}_{b}|\mu, \tau\rangle=\sqrt{\gamma} \ell_{\mathrm{Pl}} \operatorname{sgn}(\mu) \sqrt{|\tau| \mid} \mu, \tau\right\rangle .
$$

Since the operator is quantized directly, the eigenvalues are just those which one would expect from the classical expression (4) of $\omega_{b}$.

The properties of co-triad operators suggest that the lines $\mu=0$ and $\tau=0$ have very different features also in quantum geometry. No quantum effects are manifested at $\mu=0$ which corresponds to the classical horizon. Near and on the line $\tau=0$ which represents the singularity, on the other hand, quantum effects are large. In particular, they remove the classical singular behaviour of the co-triad. (Similar results have been obtained in [31] using almost periodic functions in the ADM setting and in [32] using coherent states.) We emphasize, however, that even in homogeneous models, the ultimate test as to whether or not a singularity persists upon quantization can only come from studying the dynamics. The key questions are as follows: Is quantum dynamics well defined and deterministic across the classical singularity? And, does this come about without significant quantum corrections to geometry near the horizon? Only quantum dynamics will tell us if the indications provided by the properties of the co-triad operators are borne out.

\section{Quantum dynamics}

This section is divided into three parts. In the first we present the strategy, in the second we obtain the quantum Hamiltonian constraint and in the third we use it to discuss the consequent resolution of the classical singularity.

\subsection{Strategy}

To bring out the similarities and differences between the reduced model and the full theory, it is instructive to begin with the Hamiltonian constraint (15) of the full theory:

$$
\mathcal{C}_{\text {Ham }}=\int \mathrm{d}^{3} x N e^{-1}\left[\epsilon_{i j k} E^{a i} E^{b j} F_{a b}^{k}-2\left(1+\gamma^{2}\right) E_{i}^{a} E_{j}^{b} K_{[a}^{i} K_{b]}^{j}\right] .
$$

We begin by noting some simplifications that arise because of spatial homogeneity. Recall that the connection $A$ is related to the spin connection $\Gamma$ defined by the triad and the extrinsic curvature $K$ via $A=\Gamma+\gamma K$. As remarked in section 2.1, symmetries of the model imply that $\Gamma$ is the standard magnetic monopole connection; it does not depend on the phase space point under consideration. From its expression (6), it follows that its curvature $\Omega$ is given by

$$
\Omega=-\sin \vartheta \tau_{3} \mathrm{~d} \vartheta \wedge \mathrm{d} \varphi
$$

Since it is a ' $c$-number', it can be trivially taken over to quantum theory. Now, the curvature $F$ of the full connection $A$ can be expanded out as

$F_{a b}=2 \partial_{[a} A_{b]}+\left[A_{a}, A_{b}\right]=\Omega_{a b}+2 \gamma \partial_{[a} K_{b]}+\gamma^{2}\left[K_{a}, K_{b}\right]+\gamma\left[\Gamma_{a}, K_{b}\right]-\gamma\left[\Gamma_{b}, K_{a}\right]$.

Using expressions (6), (7) and (3) of $\Gamma, K$ and $E$, it is straightforward to verify that

$$
[\Gamma, \gamma K]=b \tau_{2} \cos \vartheta \mathrm{d} \vartheta \wedge \mathrm{d} \varphi=-\gamma \mathrm{d} K, \quad \epsilon_{i j k}\left(\partial_{[a} K_{b]}^{i}\right) E_{j}^{a} E_{k}^{b}=0 .
$$


These equations now imply that the integrand of the Hamiltonian constraint can be written simply as ${ }^{11}$

$\mathcal{C}_{\mathrm{Ham}}(x)=e^{-1} E^{a i} E^{b j}\left(\epsilon_{i j k} F_{a b}^{k}-2\left(1+\gamma^{2}\right) K_{[a}^{i} K_{b]}^{j}\right)=e^{-1} E^{a i} E^{b j}\left(\epsilon_{i j k} \Omega_{a b}^{k}-2 K_{[a i} K_{b] j}\right)$.

Since $\Omega$ is constant on the entire phase space, the non-trivial part of the Hamiltonian constraint is thus contained just in a term quadratic in extrinsic curvature.

To pass to the quantum theory, we have to express the right-hand side in terms of holonomies and triads. There are two possible avenues.

(i) Using (36), the extrinsic curvature terms in the constraint function $\mathcal{C}_{\text {Ham }}(x)$ can be written in terms of curvatures $F$ and $\Omega$ so that we have

$$
\mathcal{C}_{\mathrm{Ham}}(x)=\frac{1}{\gamma^{2}} \epsilon_{i j k} e^{-1} E^{a i} E^{b j}\left[\left(1+\gamma^{2}\right) \Omega_{a b}^{k}-F_{a b}^{k}\right] .
$$

The idea now is to use the discussion of co-triads in section 3.2 to obtain the operator analogue of $\epsilon_{i j k} e^{-1} E^{a i} E^{b j}$, carry $\Omega$ to quantum theory trivially and use holonomies to express the field strength $F$ as in the full theory.

(ii) Alternatively, since we have completely fixed the gauge freedom to perform internal $S U$ (2) rotations, we can regard $K$ itself as a connection. It is a direct analogue of the connection $A$ in the spatially flat cosmologies where $\Gamma$ vanishes. Therefore, we will denote its curvature by ${ }^{o} F ;{ }^{o} F=\mathrm{d} K+[K, K]$. Then, the constraint functional can also be expressed as

$$
\mathcal{C}_{\mathrm{Ham}}(x)=\frac{1}{\gamma^{2}} \epsilon_{i j k} e^{-1} E^{a i} E^{b j}\left[\gamma^{2} \Omega_{a b}^{k}-{ }^{o} F_{a b}^{k}\right]
$$

Now, one can again use the discussion of co-triads in section 3.2 to obtain the operator analogue of $\epsilon_{i j k} e^{-1} E^{a i} E^{b j}$, carry $\Omega$ to quantum theory trivially and use holonomies to express the field strength ${ }^{o} F$. However, now holonomies have to be constructed using the connection $K$ rather than $A$. Since our Hilbert space $\mathcal{H}$ is built from holonomies of $A$, at first it seems difficult to express holonomies of $K$ as operators on $\mathcal{H}$. However, because of spatial homogeneity, we can calculate $\mathcal{C}_{\text {Ham }}(x)$ at any point. Let us choose a point on the equator and use holonomies along the three sets of curves introduced in (20)-(22). Using expressions (2) and (7) of $A$ and $K$, it follows that along these curves the holonomies of $A$ and $K$ are equal! Therefore, the required holonomies of $K$ can indeed be expressed as operators on $\mathcal{H}$.

Both these strategies are viable and, since they begin with exact expressions (38) and (39) of classical constraints, the difference in the resulting operators is just a quantization ambiguity. The first strategy is more natural in the sense that it does not require the introduction of a new connection. On the other hand, since the new connection $K$ is the direct analogue of the connection used in spatially flat cosmologies (in particular in [9]), to facilitate comparisons, the second strategy has been used in general, homogeneous models [8,33]. For definiteness, we will use the second strategy in the main discussion and comment at the end on how the first strategy modifies the final constraint operator. The analysis of the resolution of the singularity can be carried out with either methods and the conclusion is the same.

\footnotetext{
${ }^{11}$ This simplification has a natural origin. Because of (36), the right-hand side is just $\epsilon_{i j k} E^{a i} E^{b j+} F_{a b}^{k}$, where ${ }^{+} F$ is
} the curvature of the self-dual connection ${ }^{+} A=\Gamma+\mathrm{i} K$. 


\subsection{Quantum Hamiltonian constraint}

Starting from the classical Hamiltonian constraint

$$
\mathcal{C}_{\mathrm{Ham}}=\frac{1}{\gamma^{2}} \int \mathrm{d}^{3} x \epsilon_{i j k} e^{-1} E^{a i} E^{b j}\left[\gamma^{2} \Omega_{a b}^{k}-{ }^{o} F_{a b}^{k}\right]
$$

where the integral is taken over the elementary cell, we wish to pass to the quantum operator. The overall strategy is the same as in $[8,9,33]$ and the subtleties are discussed in detail in [9]. Therefore, here we will present only the main steps and comment on differences from the isotropic case treated in [9].

In the isotropic case, the elementary cell is a cube of length $L_{o}$ with respect to the fiducial metric. To express the curvature of the connection and the co-triad operator in the Hamiltonian constraint, one uses holonomies along the curves of variable length $\mu_{o} L_{o}$ along the edges of this cell. For each value of $\mu_{o}$, one obtains a quantum constraint operator $\hat{\mathcal{C}}_{\mathrm{Ham}}^{\left(\mu_{o}\right)}$. As discussed in [9], considerations from the full theory imply that $\mu_{o}$ should not be regarded as a regulator; rather, the $\mu_{o}$-dependence of this operator should be regarded as a quantization ambiguity. This ambiguity could not be fixed within the reduced model itself. But by making an appeal to results from the full theory, one can fix the value of $\mu_{o}$ using the minimum eigenvalue of the area operator in the full theory. (Recall that area enclosed by a loop enters while expressing curvature in terms of holonomies.)

In the present, Kantowski-Sachs model, the overall procedure is the same but minor adjustments are necessary because of lack of isotropy. Since $\mathbb{S}^{2}$ is compact, our elementary cell $\mathbb{S}^{2} \times \mathcal{I}$ has a geometric edge only along the $\mathcal{I}$-direction. As in section 3.1 , we will supplement it with edges along a longitude and the equator of $\mathbb{S}^{2}$. Holonomies along these three edges are then given by (20)-(22). Now consider curves of length $L_{o} \delta$ along $\mathcal{I}$ and of length $\delta$ each along the equator and the longitude of $\mathbb{S}^{2}$. Then, the co-triad function in the Hamiltonian (40) can be expressed as

$$
\epsilon_{i j k} \tau^{i} e^{-1} E^{a j} E^{b k}=-2\left(8 \pi \gamma G \mathcal{L}_{(k)}\right)^{-1} \epsilon^{a b c o} \omega_{c}^{k} h_{k}^{(\delta)}\left\{h_{k}^{(\delta)-1}, V\right\},
$$

where on the right-hand side the index $k$ runs over $x, \vartheta, \varphi$ and is summed over, $h_{k}$ is the holonomy along the edge $k$, and $\mathcal{L}_{x}=L_{o} \delta$ and $\mathcal{L}_{\vartheta}=\mathcal{L}_{\varphi}=\delta$. Note that this is an exact equality for any choice of $\delta$. Components of the curvature ${ }^{o} F$ can also be expressed in terms of these holonomies. Set

$$
h_{i j}^{(\delta)}=h_{i}^{(\delta)} h_{j}^{(\delta)}\left(h_{i}^{(\delta)}\right)^{-1}\left(h_{j}^{(\delta)}\right)^{-1}
$$

where $i, j, k$ run over $x, \vartheta, \varphi$. Then, using (20)-(22) it is straightforward to verify that

$$
{ }^{o} F_{a b}^{i}(x) \tau_{i}=\frac{{ }^{o} \omega_{a}^{i o} \omega_{b}^{j}}{\mathcal{A}_{(i j)}}\left(h_{i j}^{\mathcal{A}_{(i j)}}-1\right)+O\left(\left(b^{2}+c^{2}\right)^{3 / 2} \sqrt{\mathcal{A}}\right),
$$

where $\mathcal{A}_{x \vartheta}=\delta^{2} L_{o}=\mathcal{A}_{x \varphi}$ and $\mathcal{A}_{\vartheta \varphi}=\delta^{2}$. For ${ }^{o} F_{x, \vartheta}$ and ${ }^{o} F_{x, \varphi}$, (43) is the standard geometric relation between holonomies around closed loops, the area they enclose and curvature. For ${ }^{o} F_{\vartheta, \varphi}$, on the other hand, while (43) continues to hold, the standard geometrical interpretation is no longer available because the edges of length $\delta$ along the equator and a longitude fail to form a closed loop. Nonetheless, the standard relation is meaningfully extended because of spatial homogeneity, $\delta^{2}$ playing the role of the 'effective area' $\mathcal{A}_{\vartheta, \varphi}$.

We now have all the ingredients, ${ }^{\circ} F, \Omega$ and the triad components, to rewrite the classical constraint (40) in a way which is suitable for quantization. For the ${ }^{o} F$-term in (40), 
we obtain

$$
\begin{aligned}
-\gamma^{-2} \int \mathrm{d}^{3} x & \epsilon_{i j k}{ }^{o} F_{a b}^{i} e^{-1} E^{a j} E^{b k}=2 \gamma^{-2} \int \mathrm{d}^{3} x \operatorname{Tr}\left({ }^{o} F_{a b}^{i} \tau_{i} \epsilon_{j k l} e^{-1} E^{a j} E^{b k} \tau^{l}\right) \\
= & -4\left(8 \pi \gamma^{3} G\right)^{-1} \int \mathrm{d}^{3} x \operatorname{det}^{o} \omega \operatorname{Tr}\left(\epsilon^{i j k} \mathcal{A}_{(i j)}^{-1}\left(h_{i j}^{(\delta)}-1\right) \mathcal{L}_{(k)}^{-1} h_{k}^{(\delta)}\left\{h_{k}^{(\delta)-1}, V\right\}\right) \\
& +O\left(\left(b^{2}+c^{2}\right)^{3 / 2} \delta\right) \\
= & {\left[-16 \pi\left(8 \pi \gamma^{3} G \delta^{3}\right)^{-1} \sum_{i j k} \epsilon^{i j k} \operatorname{Tr}\left(h_{i j}^{(\delta)} h_{k}^{(\delta)}\left\{h_{k}^{(\delta)}, V\right\}\right)\right]+O\left(\left(b^{2}+c^{2}\right)^{3 / 2} \delta\right) . }
\end{aligned}
$$

Next, using $\Omega=-\sin \vartheta \mathrm{d} \vartheta \wedge \mathrm{d} \varphi \tau_{3}$, the $\Omega$ term in (40) becomes

$$
\begin{aligned}
\int \mathrm{d}^{3} x \epsilon_{i j k} \Omega_{a b}^{i} e^{-1} E^{a j} E^{b k} & =-2 \int \mathrm{d}^{3} x \operatorname{Tr}\left(\Omega_{a b}^{i} \tau_{i} \epsilon_{j k l} e^{-1} E^{a l} E^{b k} \tau^{l}\right) \\
& =-8(8 \pi \gamma G \delta)^{-1} \int \mathrm{d}^{3} x \sin \vartheta \operatorname{Tr}\left(\tau_{3} \mathcal{L}_{x}^{-1} h_{x}^{(\delta)}\left\{h_{x}^{(\delta)-1}, V\right\}\right) \\
& =-32 \pi(8 \pi \gamma G \delta)^{-1} \operatorname{Tr}\left(\tau_{3} h_{x}^{(\delta)}\left\{h_{x}^{(\delta)-1}, V\right\}\right) .
\end{aligned}
$$

Set

$$
\begin{array}{r}
C^{(\delta)}=\left[-2\left(\gamma^{3} G \delta^{3}\right)^{-1} \sum_{i j k} \epsilon^{i j k} \operatorname{Tr}\left(h_{i j}^{(\delta)} h_{k}^{(\delta)}\left\{h_{k}^{(\delta)}, V\right\}\right)\right] \\
-\left[-4(\gamma G \delta)^{-1} \operatorname{Tr}\left(\tau_{3} h_{x}^{(\delta)}\left\{h_{x}^{(\delta)-1}, V\right\}\right)\right] .
\end{array}
$$

Then the classical Hamiltonian constraint is given by

$$
\mathcal{C}_{\text {Ham }}=\lim _{\delta \rightarrow 0} C^{(\delta)} .
$$

Since all terms in $C^{\delta}$ are expressed purely in terms of our elementary variables-holonomies and triads-which have direct operator analogues, passage to quantum theory is now straightforward. We obtain

$$
\begin{aligned}
\hat{C}^{(\delta)}= & 2 \mathrm{i}\left(\gamma^{3} \delta^{3} \ell_{\mathrm{Pl}}^{2}\right)^{-1} \operatorname{Tr}\left(\sum_{i j k} \epsilon^{i j k} \hat{h}_{i}^{(\delta)} \hat{h}_{j}^{(\delta)} \hat{h}_{i}^{(\delta)-1} \hat{h}_{j}^{(\delta)-1} \hat{h}_{k}^{(\delta)}\left[\hat{h}_{k}^{(\delta)-1}, \hat{V}\right]\right. \\
& \left.+2 \gamma^{2} \delta^{2} \tau_{3} \hat{h}_{x}^{(\delta)}\left[\hat{h}_{x}^{(\delta)-1}, \hat{V}\right]\right) \\
= & 4 \mathrm{i}\left(\gamma^{3} \delta^{3} \ell_{\mathrm{Pl}}^{2}\right)^{-1}\left(8 \sin \frac{\delta b}{2} \cos \frac{\delta b}{2} \sin \frac{\delta c}{2} \cos \frac{\delta c}{2}\left(\sin \frac{\delta b}{2} \hat{V} \cos \frac{\delta b}{2}-\cos \frac{\delta b}{2} \hat{V} \sin \frac{\delta b}{2}\right)\right. \\
& \left.+\left(4 \sin ^{2} \frac{\delta b}{2} \cos ^{2} \frac{\delta b}{2}+\gamma^{2} \delta^{2}\right)\left(\sin \frac{\delta c}{2} \hat{V} \cos \frac{\delta c}{2}-\cos \frac{\delta c}{2} \hat{V} \sin \frac{\delta c}{2}\right)\right)
\end{aligned}
$$

(This is a special case of equation (26) in [33].)

The action of this operator on the eigenstates $|\mu, \tau\rangle$ of $\hat{p}_{b}$ and $\hat{p}_{c}$ is given by

$$
\begin{aligned}
\hat{C}^{(\delta)}|\mu, \tau\rangle= & \left(2 \gamma^{3} \delta^{3} \ell_{\mathrm{Pl}}^{2}\right)^{-1}\left[2\left(V_{\mu+\delta, \tau}-V_{\mu-\delta, \tau}\right)(|\mu+2 \delta, \tau+2 \delta\rangle-|\mu+2 \delta, \tau-2 \delta\rangle\right. \\
& -|\mu-2 \delta, \tau+2 \delta\rangle+|\mu-2 \delta, \tau-2 \delta\rangle) \\
& \left.+\left(V_{\mu, \tau+\delta}-V_{\mu, \tau-\delta}\right)\left(|\mu+4 \delta, \tau\rangle-2\left(1+2 \gamma^{2} \delta^{2}\right)|\mu, \tau\rangle+|\mu-4 \delta, \tau\rangle\right)\right] .
\end{aligned}
$$

However, while the classical constraint is a real function on the kinematical phase space $\Gamma, \hat{C}^{\delta}$ fails to be self-adjoint on the kinematical Hilbert space $\mathcal{H}$. We therefore need to add to 
it its Hermitian adjoint ${ }^{12}$. The result is an operator $\hat{C}_{\text {grav }}^{(\delta)}:=\frac{1}{2}\left(\hat{C}^{(\delta)}+\hat{C}^{(\delta) \dagger}\right)$ given by

$$
\begin{aligned}
\hat{C}_{\text {grav }}^{(\delta)}|\mu, \tau\rangle= & \left(2 \gamma^{3} \delta^{3} \ell_{\mathrm{Pl}}^{2}\right)^{-1}\left[\left(V_{\mu+\delta, \tau}-V_{\mu-\delta, \tau}+V_{\mu+3 \delta, \tau+2 \delta}-V_{\mu+\delta, \tau+2 \delta}\right)|\mu+2 \delta, \tau+2 \delta\rangle\right. \\
& -\left(V_{\mu+\delta, \tau}-V_{\mu-\delta, \tau}+V_{\mu+3 \delta, \tau-2 \delta}-V_{\mu+\delta, \tau-2 \delta}\right)|\mu+2 \delta, \tau-2 \delta\rangle \\
& -\left(V_{\mu+\delta, \tau}-V_{\mu-\delta, \tau}+V_{\mu-\delta, \tau+2 \delta}-V_{\mu-3 \delta, \tau+2 \delta}\right)|\mu-2 \delta, \tau+2 \delta\rangle \\
& +\left(V_{\mu+\delta, \tau}-V_{\mu-\delta, \tau}+V_{\mu-\delta, \tau-2 \delta}-V_{\mu-3 \delta, \tau-2 \delta}\right)|\mu-2 \delta, \tau-2 \delta\rangle \\
& +\frac{1}{2}\left(V_{\mu, \tau+\delta}-V_{\mu, \tau-\delta}+V_{\mu+4 \delta, \tau+\delta}-V_{\mu+4 \delta, \tau-\delta}\right)|\mu+4 \delta, \tau\rangle \\
& -\left(1+2 \gamma^{2} \delta^{2}\right)\left(V_{\mu, \tau+\delta}-V_{\mu, \tau-\delta}\right)|\mu, \tau\rangle \\
& \left.+\frac{1}{2}\left(V_{\mu, \tau+\delta}-V_{\mu, \tau-\delta}+V_{\mu-4 \delta, \tau+\delta}-V_{\mu-4 \delta, \tau-\delta}\right)|\mu-4 \delta, \tau\rangle\right] .
\end{aligned}
$$

Physical states in quantum theory are those which are symmetric under the 'parity operator' $\hat{\Pi}_{b}$ and lie in the kernel of the operator $\hat{C}_{\text {grav }}^{(\delta)}$. That is, the only non-trivial quantum Einstein equations are

$$
\left(\Psi \mid \hat{\Pi}_{b}=0, \quad\left(\Psi \mid \hat{C}_{\text {grav }}^{(\delta)}=0,\right.\right.
$$

where $\left(\Psi \mid\right.$ is an element of the dual $\mathrm{Cy}_{\mathrm{KS}}^{\star}$ of the space $\mathrm{Cyl}_{\mathrm{KS}}$ of finite linear combinations of almost periodic functions of $b, c$. Let us expand ( $\Psi \mid$ using eigenbras $\left(\mu, \tau \mid\right.$ (in $\left.\mathrm{Cyl}_{\mathrm{KS}}^{\star}\right)$ of the triad operators:

$$
\left(\Psi \mid=\sum_{\mu, \tau} \psi_{\mu, \tau}(\mu, \tau \mid .\right.
$$

Then, $\psi_{\mu, \tau}$ can be regarded as wavefunctions in the triad (or Riemannian geometry) representation. To exhibit the action of $\hat{C}_{\text {grav }}^{(\delta)}$ on $\psi_{\mu, \tau}$, we are led to separate the cases $\mu \geqslant 4 \delta$ from $\mu<4 \delta$ because of the absolute value $|\mu|$ in the volume eigenvalues. For $\mu \geqslant 4 \delta$, the quantum Einstein equation (53) becomes

$$
\begin{aligned}
\hat{C}_{\text {grav }}^{(\delta)} \psi_{\mu, \tau}= & 2 \delta(\sqrt{|\tau+2 \delta|}+\sqrt{|\tau|})\left(\psi_{\mu+2 \delta, \tau+2 \delta}-\psi_{\mu-2 \delta, \tau+2 \delta}\right) \\
& +(\sqrt{|\tau+\delta|}-\sqrt{|\tau-\delta|})\left((\mu+2 \delta) \psi_{\mu+4 \delta, \tau}-\left(1+2 \gamma^{2} \delta^{2}\right) \mu \psi_{\mu, \tau}\right. \\
& \left.+(\mu-2 \delta) \psi_{\mu-4 \delta, \tau}\right)+2 \delta(\sqrt{|\tau-2 \delta|}+\sqrt{|\tau|})\left(\psi_{\mu-2 \delta, \tau-2 \delta}-\psi_{\mu+2 \delta, \tau-2 \delta}\right) \\
= & 0 .
\end{aligned}
$$

For $\mu=0$, we have

$\sqrt{|\tau+2 \delta|} \psi_{2 \delta, \tau+2 \delta}+(\sqrt{|\tau+\delta|}-\sqrt{|\tau-\delta|}) \psi_{4 \delta, \tau}-\sqrt{|\tau-2 \delta|} \psi_{2 \delta, \tau-2 \delta}=0$

for $\mu=\delta$, we have

$$
\begin{aligned}
2(\sqrt{|\tau+2 \delta|}+ & \sqrt{|\tau|}) \psi_{3 \delta, \tau+2 \delta}+2(\sqrt{|\tau+2 \delta|}-\sqrt{|\tau|}) \psi_{\delta, \tau+2 \delta} \\
& +(\sqrt{|\tau+\delta|}-\sqrt{|\tau-\delta|})\left(3 \psi_{5 \delta, \tau}+2 \psi_{3 \delta, \tau}-\left(1+2 \gamma^{2} \delta^{2}\right) \psi_{\delta, \tau}\right) \\
& -2(\sqrt{|\tau-2 \delta|}+\sqrt{|\tau|}) \psi_{3 \delta, \tau-2 \delta}-2(\sqrt{|\tau-2 \delta|}-\sqrt{|\tau|}) \psi_{\delta, \tau-2 \delta}=0,
\end{aligned}
$$

for $\mu=2 \delta$, we have

$$
\begin{aligned}
(\sqrt{|\tau+2 \delta|}+\sqrt{|\tau|}) \psi_{4 \delta, \tau+2 \delta}-\sqrt{|\tau|} \psi_{0, \tau+2 \delta} \\
+(\sqrt{|\tau+\delta|}-\sqrt{|\tau-\delta|})\left(2 \psi_{6 \delta, \tau}+\psi_{2 \delta, \tau}-\left(1+2 \gamma^{2} \delta^{2}\right) \psi_{2 \delta, \tau}\right) \\
\quad-(\sqrt{|\tau-2 \delta|}+\sqrt{|\tau|}) \psi_{4 \delta, \tau-2 \delta}+\sqrt{|\tau|} \psi_{0, \tau-2 \delta}=0
\end{aligned}
$$

\footnotetext{
12 Although the self-adjoint form of the Hamiltonian constraint was discussed briefly in [35], this point was ignored in the detailed treatment of [9]. A detailed treatment of the self-adjoint constraint and its semi-classical implications can be found in $[27,36]$. It is used in a crucial way to obtain the physical Hilbert space in [10].
} 
and for $\mu=3 \delta$, we have

$$
\begin{aligned}
2(\sqrt{|\tau+2 \delta|}+ & \sqrt{|\tau|})\left(\psi_{5 \delta, \tau+2 \delta}-\psi_{\delta, \tau+2 \delta}\right) \\
& +(\sqrt{|\tau+\delta|}-\sqrt{|\tau-\delta|})\left(5 \psi_{7 \delta, \tau}+2 \psi_{\delta, \tau}-3\left(1+2 \gamma^{2} \delta^{2}\right) \psi_{3 \delta, \tau}\right) \\
& -2(\sqrt{|\tau-2 \delta|}+\sqrt{|\tau|})\left(\psi_{5 \delta, \tau-2 \delta}-\psi_{\delta, \tau-2 \delta}\right)=0 .
\end{aligned}
$$

For values of $\mu$ not an integer multiple of $\delta$, one can derive similar expressions, but they will not be used in the following. As remarked above, strictly speaking, the role of (55) is only to select physical wavefunctions. However, intuitively it can also be thought of as the quantum evolution equation. Recall that $p_{c}$ can be regarded as an internal time in the classical theory of the model. Therefore, in the quantum theory, one may regard $\tau$ as internal time. Then, (55) can be interpreted as the quantum Einstein equation, which evolves the wavefunctions in discrete steps of magnitude $2 \delta$ along $\tau$.

So far, the parameter $\delta$ - and hence the size of the 'time step'-is arbitrary. In the classical theory, we recover the Hamiltonian constraint only in the limit $\delta$ goes to zero. This is because, while expression (4) is exact for all loops, curvature ${ }^{\circ} F$ can be expressed in terms of holonomies only in the limit in which the areas of loops shrink to zero (see (43)). In quantum theory, however, the straightforward $\operatorname{limit}_{\lim } \operatorname{lo}_{\delta \rightarrow 0} \hat{C}^{(\delta)}$ diverges (for the same reasons as in the isotropic case [9]). As in quantum cosmology [9], the viewpoint is that this occurs because the limit ignores the quantum nature of geometry, i.e., the fact that in full quantum geometry, the area operator has a minimum non-zero eigenvalue. The 'correct' quantization of the Hamiltonian constraint $\mathcal{C}_{\text {Ham }}$ has to take in to account the quantum nature of geometry.

To do so, note first that the holonomies used in the expression of ${ }^{o} F$ define quantum states with $\mu=\delta$ and $\tau=\delta$. Using these states, we can calculate the quantum area of each of the three faces of the elementary cell $\mathbb{S}^{2} \times \mathcal{I}$, enclosed by the three curves—one along $x$, one along a longitude and one along the equator of $\mathbb{S}^{2}$. The area operators defined by faces $S_{x, \vartheta}, S_{x, \varphi}$ and $S_{\vartheta, \varphi}$ are, respectively, $2 \pi\left|\hat{p}_{b}\right|, 2 \pi\left|\hat{p}_{b}\right|$ and $\pi\left|\hat{p}_{c}\right|$ (see (14)). The quantum geometry states defined by the three holonomies are eigenstates of these area operators. Furthermore, they have the same eigenvalue: $\pi \gamma \delta \ell_{\mathrm{Pl}}^{2}$. Now, in the full theory, we know that the area operator has a minimum non-zero eigenvalue, $a_{o}=2 \sqrt{3} \pi \gamma \ell_{\mathrm{Pl}}^{2}$. The viewpoint, as in [9], is that in the calculation of the field strength ${ }^{o} F$, it is physically inappropriate to try to use surfaces of arbitrarily small areas. The best one can do is to shrink the area of the loop till it attains this quantum minimum $a_{o}$. This implies that we should set $\delta=2 \sqrt{3}$. To summarize, by using input from the full theory, we conclude that the quantum Hamiltonian constraint is given by $\hat{C}^{\left(\delta_{o}\right)}$ with $\delta_{o}=2 \sqrt{3} \cdot{ }^{13}$

\section{Remarks.}

(i) Note that the character of the difference equation changes depending on whether $\mu \geqslant 4 \delta$ or $\mu<4 \delta$. In particular, for $\mu \geqslant 4 \delta$, the knowledge of the wavefunction at 'times' $\tau+2 \delta$ and $\tau$ determines only the combination $\psi_{\mu-2 \delta, \tau-2 \delta}-\psi_{\mu+2 \delta, \tau-2 \delta}$ at 'time' $\tau-2 \delta$ via (55). On the other hand, for $\mu=0$ the wavefunction at $\tau+2 \delta$ and $\tau$ determines $\psi_{2 \delta, \tau+2 \delta}$ completely via (56). We will return to these differences in section 4.3.

(ii) As discussed in $[9,34,37]$ in detail, one can recover the Wheeler-DeWitt equation with a specific factor ordering by taking a systematic limit of the quantum Einstein equation. In the present model, the difference equation reduces to the following differential equation

\footnotetext{
${ }^{13}$ For a detailed discussion of this point, including a comparison with the situation in full loop quantum gravity and
} the precise sense in which the $\delta$-ambiguity is analogous to a factor ordering ambiguity there, see [9]. 
for the wavefunction $\Psi\left(p_{b}, p_{c}\right)=\psi_{2 p_{b} / \gamma \ell_{\mathrm{P} \mid}^{2}, p_{c} / \gamma \ell_{\mathrm{P} \mid}^{2}}$ on the classical minisuperspace $\mathcal{M}$ :

$$
16 \ell_{\mathrm{Pl}}^{4}\left(\sqrt{p_{c}} \frac{\partial^{2} \Psi}{\partial p_{b} \partial p_{c}}+\frac{p_{b}}{4 \sqrt{p_{c}}} \frac{\partial^{2} \Psi}{\partial p_{b}^{2}}+\frac{1}{2 \sqrt{p_{c}}} \frac{\partial \Psi}{\partial p_{b}}\right)-4 \frac{p_{b}}{\sqrt{p_{c}}} \Psi=0 .
$$

For $\mu \gg \delta, \tau \gg \delta$, solutions of the discrete equation (55) can be approximated by those of this Wheeler-DeWitt equation in a precise sense. In essence, this WheelerDeWitt equation is obtained from (55) by ignoring quantum geometry effects, i.e., by an appropriate $\delta \rightarrow 0$ limit. For the approximation by a differential equation, higher derivatives of the wavefunction $\psi$ must be sufficiently small. Thus, the limit $\delta \rightarrow 0$ for equation (55) exists only under additional assumptions on the wavefunction. It does not exist for the operator $\hat{C}_{\text {grav }}^{(\delta)}$ by itself.

\subsection{Absence of singularity}

Recall that the classical singularity occurs at $p_{c}=0$. The Wheeler-DeWitt equation (60) is manifestly singular there. One can multiply it by $\sqrt{p_{c}}$ and define a new 'internal time', $\bar{p}_{c}=\ln p_{c}$, and obtain a regular equation in variables $p_{b}, \bar{p}_{c}$. However, since $\bar{p}_{c} \rightarrow-\infty$ at the singularity, this regular equation does not let us evolve across the singularity. Thus, the overall situation is the same as in the isotropic model.

To obtain the Wheeler-DeWitt equation from the 'fundamental' difference equation (55), we had to ignore quantum geometry effects. Thus, the key question is whether this failure of the Wheeler-DeWitt equation is an artefact of the approximation used. Can quantum geometry effects make a qualitative difference as they do in the isotropic model? We will now argue that the answer is in the affirmative.

Let us analyse the 'evolution' given by the difference equation (55). Does this evolution stop at $\tau=0$ ? As in the isotropic case [7,35], we will use the quantum constraint (55) as a recurrence relation starting at positive $\tau$ and evolve towards smaller values. However, now a new twist arises because for generic values $\mu \geqslant 4 \delta$, (55) determines only the difference $\psi_{\mu+2 \delta, \tau-2 \delta}-\psi_{\mu-2 \delta, \tau-2 \delta}$ as a function of the initial values of the wavefunction. Therefore, the quantum Einstein equation has to be supplemented with an appropriate boundary condition. From (55) which holds for $\mu \geqslant 4 \delta$, one might conclude that, even if we restrict ourselves to the 'lattice' $\mu=n \delta$, one would have to specify the wavefunction at $\mu=0, \delta, 2 \delta, 3 \delta$ at each 'time step'. However, as remarked at the end of section 4.2, the form of the difference equations for $\mu<4 \delta$ is different. An examination of (56) and (57) reveals that it is in fact sufficient to specify the wavefunction $\psi_{\mu, \tau}$ just at $\mu=0$ and $\mu=\delta$ at each 'time step'. This is a mathematically viable choice of the boundary condition and heuristically it corresponds to providing data at the 'horizon'. It will turn out that the issue of the resolution of singularity is insensitive to the precise choice of the boundary condition. We will therefore postpone the discussion of physically appropriate choices until after the main discussion.

Let us then start at some finite positive $\tau$ and carry out a backward evolution using (55). In contrast to the Wheeler-DeWitt equation (60), the coefficients in the difference equation (55) are always regular. At first, one might think that this regularity by itself would be sufficient to ensure that the evolution would be well defined across the singularity. Note however that this need not be the case, because the backward evolution continues only as long as the coefficient of the wavefunction at $\tau-2 \delta$ is non-zero. The key question therefore is whether this coefficient vanishes. The issue is subtle. For instance, the coefficients in the non-self-adjoint operator $\left(\hat{C}^{\delta}\right)^{\dagger}$ are also non-singular. However, if one uses it in place of $\hat{C}_{\text {Ham }}^{\delta}$, one finds that the coefficient vanishes and one cannot evolve across the singularity. 
For our quantum Einstein equation (55), this coefficient is given by $\sqrt{|\tau-2 \delta|}+\sqrt{|\tau|}$. By inspection, it never vanishes. Therefore, the backward quantum evolution remains well defined and determines the wavefunction not only for $\tau>0$ but also in the new region with $\tau \leqslant 0$. In this precise sense, the classical black hole singularity can be traversed using quantum evolution and thus ceases to be a boundary of spacetime.

Next, recall from section 4.1 that we could have begun with expression (38) of the Hamiltonian constraint in the classical theory and then proceeded with quantization. What would be the status of the singularity resolution with this choice? The procedure to construct the quantum Hamiltonian constraint would be identical. However, the final result would be slightly different: $\gamma^{2}$ in (55) would be replaced by $1+\gamma^{2}$. That is, in the new quantum Einstein equation, the coefficient of $\psi_{\mu}, \tau$ in (55) would be modified and the equation would become

$$
\begin{aligned}
2 \delta(\sqrt{|\tau+2 \delta|}+ & \sqrt{|\tau|})\left(\psi_{\mu+2 \delta, \tau+2 \delta}-\psi_{\mu-2 \delta, \tau+2 \delta}\right) \\
& +(\sqrt{|\tau+\delta|}-\sqrt{|\tau-\delta|})\left((\mu+2 \delta) \psi_{\mu+4 \delta, \tau}-\left(1+2\left(\gamma^{2}+1\right) \delta^{2}\right) \mu \psi_{\mu, \tau}\right. \\
& \left.+(\mu-2 \delta) \psi_{\mu-4 \delta, \tau}\right)+2 \delta(\sqrt{|\tau-2 \delta|}+\sqrt{|\tau|})\left(\psi_{\mu-2 \delta, \tau-2 \delta}-\psi_{\mu+2 \delta, \tau-2 \delta}\right) \\
= & 0 .
\end{aligned}
$$

(The same replacement holds for $\mu<4 \delta$.) Since the coefficient of $\psi_{\mu-2 \delta, \tau-2 \delta}-\psi_{\mu+2 \delta, \tau-2 \delta}$ is unaltered, one would again be able to 'evolve' across the singularity. Thus, the conclusion is robust within quantum geometry ${ }^{14}$.

To conclude, we will briefly return to the questions of the boundary condition that are needed to make the evolution well defined even away from the singularity. As noted earlier, in the backward evolution given by (56), $\psi_{2 \delta, \tau_{o}}$ is determined by values of the wavefunction at $\tau>\tau_{o}$, so long as $\tau_{o} \neq 0$. Furthermore, (55) then determines the wavefunction at $\mu=(2+4 n) \delta$ for all $n \in \mathbb{N}$ and at that $\tau_{o}$. One could now use pre-classicality arguments [38] (i.e. require that the wavefunction not oscillate on small scales) at large $\mu$ to choose the boundary values, eliminating entirely the need for specifying boundary conditions. While this could be reasonable in semi-classical regimes, for small $|\tau|$ it would be questionable to use preclassicality in the $\mu$-direction even if a pre-classical solution exist (which is not guaranteed, see e.g. the analysis in [39, 40]). More importantly, the slice $\tau=0$ is special because $\psi_{2 \delta, 0}$ is not determined through (56) or otherwise. At this point, condition (56) evaluated at $\tau=-2 \delta$ gives instead a condition for previous values of the wavefunction, translating into one condition for the initial values. At $\tau=0$, however, $\psi_{2 \delta, 0}$ has to be specified as boundary value in addition to $\psi_{0,0}$ because, unlike similar situations in isotropic models, it does not drop out of the evolution: it is needed in the recurrence (55) for $\mu=4 \delta, \tau=2 \delta$. There is thus no reduction in conditions on the wavefunction from the fact that $\psi_{2 \delta, 0}$ drops out of (56); a condition is simply transferred from boundary values to initial values. Note however that, even though the line $\tau=0$ (corresponding to the classical singularity) does show special behaviour, evolution does not break down there; it is only the boundary value problem which changes character.

While such a boundary value problem is mathematically well defined, the resulting theory is not necessarily physically correct, because in a physically interesting theory one would expect that, well away from the singularity, there should exist semi-classical solutions which are peaked on the classical trajectories. From figure 1 it is clear that the semi-classical solution peaked on the trajectory labelled by mass $m$ would be sharply peaked at $\tau= \pm 4 m^{2}$ on the line $\mu=0$, whence semi-classical states peaked at different classical solutions will have quite

${ }^{14}$ However, the relation between the wavefunction and the Wheeler-DeWitt approximation is less direct with this form of the constraint. 
different forms near $\mu=0$. Therefore, the theory obtained by simply fixing the wavefunction on (or near) $\mu=0$ is not likely to admit a sufficiently rich semi-classical sector. Indeed, it is not clear that any boundary condition at (or near) $\mu=0$ will be physically viable. Rather, one may have to impose the boundary condition at $\mu=\infty$, e.g., by requiring that the wavefunction should vanish there. Indeed, the form of dynamical trajectories of figure 1 implies that every semi-classical state would share this property. Moreover, the corresponding condition does hold in the closed isotropic model with a massless scalar field as source [41]. As in that case, one would expect that these semi-classical states would span a dense subspace in the physical Hilbert space. If so, requiring that the wavefunctions vanish at $\mu=\infty$ would be physically justified. Heuristics suggest that this strategy is viable for the Wheeler-DeWitt equation (60). However, detailed numerical analysis is necessary to establish that the difference equations (55) or (61) admit solutions satisfying this condition (or a suitable modification thereof) for all $\tau$ and that its imposition makes the evolution unambiguous.

\section{Discussion}

Results of the last two sections support a general scenario that has emerged from the analysis of singularities in quantum cosmology. It suggests that the classical singularity does not represent a final frontier; the physical spacetime does not end there. In the Planck regime, quantum fluctuations do indeed become so strong that the classical description breaks down. The spacetime continuum of classical general relativity is replaced by discrete quantum geometry which remains regular during the transition through what was a classical singularity. Certain similarities between the Kantowski-Sachs model analysed here and a cosmological model which has been studied in detail [10] suggest that there may be a quantum bounce to another large classical region. If this is borne out by detailed numerical calculations, one would conclude that quantum geometry in the Planck regime serves as a bridge between two large classical regions. Spacetime may be much larger than general relativity has had us believe.

However, as indicated at the end of section 4.2, significant numerical work is still needed before one can be certain that this scenario is really borne out in the model. Moreover, this is a highly simplified model. It is important to check if the qualitative conclusions remain robust as more and more realistic features are introduced. First, one should extend the analysis so that the spacetime region outside the horizon is also covered. A second and much more important challenge is incorporation of an infinite number of degrees of freedom by coupling the model, e.g., to a spherically symmetric scalar field. First steps along these lines have been taken [31, 42] and one can see that the evolution still extends beyond the classical singularity [43]. But a comprehensive treatment still remains a distant goal. Finally, since we restricted ourselves to a spacelike classical singularity, qualitative features of this singularity resolution can be valid only for a spherical collapse of uncharged fields. As explained in section 1, in more general situations the generic classical singularity is likely to be null, representing a Cauchy horizon. Therefore, although one might hope that quantum geometry effects would again extend spacetime beyond this Cauchy horizon, the detailed analysis will be significantly different.

\section{Acknowledgments}

This work was supported in part by NSF grants PHY-0090091, PHY-0354932 and PHY0456931, the Alexander von Humboldt Foundation, the C V Raman Chair of the Indian Academy of Sciences and the Eberly research funds of Penn State. 


\section{References}

[1] Misner C W 1972 Minisuperspaces Magic Without Magic, John A Wheeler Festschrift ed J R Klauder (San Francisco: Freeman)

[2] Hartle J B 1995 Spacetime quantum mechanics and the quantum mechanics of spacetime Gravitation and Quantizations: Proc. 1992 Les Houches Summer School (Amsterdam: North-Holland) (Preprint gr-qc/9304006)

[3] Hartle J B and Hawking S W 1983 The wavefunction of the universe Phys. Rev. D 28 2960-75

[4] Ashtekar A and Lewandowski L 2004 Background independent quantum gravity: a status report Class. Quantum Grav. 21 R53-R152

[5] Rovelli C 2004 Quantum Gravity (Cambridge: Cambridge University Press)

[6] Thiemann T 2004 Modern Canonical Quantum General Relativity (Cambridge: Cambridge University Press) at press Preprint gr-qc/0110034

[7] Bojowald M 2001 Absence of singularity in loop quantum cosmology Phys. Rev. Lett. 86 5227-30

[8] Bojowald M 2003 Homogeneous loop quantum cosmology Class. Quantum Grav. 20 2595-615

[9] Ashtekar A, Bojowald M and Lewandowski J 2003 Mathematical structure of loop quantum cosmology Adv. Theor. Math. Phys. 7 233-68

[10] Ashtekar A, Pawlowski T and Singh P 2005 Quantum nature of the big bang: an analytical and numerical study, in preparation

[11] Dafermos M 2003 Black hole formation from a complete regular past Preprint gr-qc/0310040

[12] Dafermos M 2003 The interior of charged black holes and the problem of uniqueness in general relativity Preprint gr-qc/0307013

[13] Horowitz G T and Myers R 1995 The value of singularities Gen. Rel. Grav. 27 915-9

[14] Marolf D 1995 Refined algebraic quantization: systems with a single constraint Preprint gr-qc/9508015

Giulini N and Marolf D 1999 On the generality of refined algebraic quantization Class. Quantum Grav. 16 2479-88

Giulini N and Marolf D 1999 A uniqueness theorem for constraint quantization Class. Quantum Grav. 16 2489-505

[15] Ashtekar A and Bojowald M 2005 Black hole evaporation: a paradigm Class. Quantum Grav. 22 3349-62

[16] Stevens C R, 't Hooft G and Whiting B F 1994 Black hole evaporation without information loss Class. Quantum Grav. 11 621-48

[17] Hayward S 2005 The disinformation problem for black holes (conference version) Preprint gr-qc/0504038

[18] Modesto L 2004 The Kantowski-Sachs space-time in loop quantum gravity Preprint gr-qc/0411032

[19] Kobayashi S and Nomizu K 1963 Foundations of Differential Geometry (New York: Wiley)

[20] Brodbeck O 1996 On symmetric gauge fields for arbitrary gauge and symmetry groups Helv. Phys. Acta 69 321-4

[21] Bojowald M and Kastrup H A 2000 Symmetry reduction for quantized diffeomorphism invariant theories of connections Class. Quantum Grav. 17 3009-43

[22] Ashtekar A and Samuel J 1991 Bianchi cosmologies: the role of spatial topology Class. Quantum Grav. 8 2191-215

[23] Kastrup H A and Thiemann T 1994 Spherically symmetric gravity as a completely integrable system Nucl. Phys. B 425 665-86

[24] Kuchař K V 1994 Geometrodynamics of Schwarzschild black holes Phys. Rev. D 50 3961-81

[25] Thiemann T 1996 Anomaly-free formulation of non-perturbative, four-dimensional Lorentzian quantum gravity Phys. Lett. B 380 257-64

Thiemann T 1998 Quantum spin dynamics (QSD) Class. Quantum Grav. 15 839-73

Thiemann T 1998 QSD V: quantum gravity as the natural regulator of matter quantum field theories Class. Quantum Grav. 15 1281-314

[26] Ashtekar A, Fairhurst S and Willis J 2003 Quantum gravity, shadow states, and quantum mechanics Class. Quantum Grav. 20 1031-62

[27] Willis J 2004 On the low-energy ramifications and a mathematical extension of loop quantum gravity PhD Dissertation Pennsylvania State University

[28] Bojowald M 2001 Inverse scale factor in isotropic quantum geometry Phys. Rev. D 64084018

[29] Bojowald M 2005 Degenerate configurations, singularities and the non-Abelian nature of loop quantum gravity Preprint gr-qc/0508118

[30] Brunnemann J and Thiemann T 2005 Unboundedness of triad-like operators in loop quantum gravity Preprint gr-qc/0505033

[31] Husain V and Winkler O 2004 Quantum resolution of black hole singularities Class. Quantum Grav. 22 L127-34 
[32] Dasgupta A 2005 Semiclassical quantisation of space-times with apparent horizons Preprint gr-qc/0505017

[33] Bojowald M, Date G and Vandersloot K 2004 Homogeneous loop quantum cosmology: the role of the spin connection Class. Quantum Grav. 21 1253-78

[34] Bojowald M and Date G 2004 Consistency conditions for fundamentally discrete theories Class. Quantum Grav. 21 121-43

[35] Bojowald M 2002 Isotropic loop quantum cosmology Class. Quantum Grav. 19 2717-41

[36] Ashtekar A, Bojowald M and Willis J 2005 Modifications to Friedmann's equation from quantum geometry, in preparation

[37] Bojowald M 2001 The semiclassical limit of loop quantum cosmology Class. Quantum Grav. 18 L109-L116

[38] Bojowald M 2001 Dynamical initial conditions in quantum cosmology Phys. Rev. Lett. 87121301

[39] Cartin D and Khanna G 2005 Absence of pre-classical solutions in Bianchi I loop quantum cosmology Phys. Rev. Lett. 94111302

[40] Date G 2005 Pre-classical solutions of the vacuum Bianchi I loop quantum cosmology Phys. Rev. D 72067301

[41] Ashtekar A, Pawlowski T and Singh P 2005 Closed cosmology and quantum geometry, in preparation

[42] Bojowald M 2004 Spherically symmetric quantum geometry: states and basic operators Class. Quantum Grav. 21 3733-53

Bojowald M and Swiderski R 2004 The volume operator in spherically symmetric quantum geometry Class. Quantum Grav. 21 4881-900

Bojowald M and Swiderski R 2005 Spherically symmetric quantum geometry: Hamiltonian constraint Preprint gr-qc/0511108

[43] Bojowald M 2005 Non-singular black holes and degrees of freedom in quantum gravity Phys. Rev. Lett. 95061301 